University of Nebraska - Lincoln

DigitalCommons@University of Nebraska - Lincoln

Robert Streubel Papers

Research Papers in Physics and Astronomy

$1-1-2015$

\title{
Magnetic soft x-ray tomography of magnetic Swiss roll architectures
}

Robert Streubel

Leibniz-Institut für Festkörper- und Werkstoffforschung Dresden, streubel@unl.edu

F. Kronast

Helmholtz-Zentrum Berlin für Materialien und Energie (HZB)

P. Fischer

Lawrence Berkeley National Laboratory

O. G. Schmidt

Leibniz-Institut für Festkörper- und Werkstoffforschung Dresden

D. Makarov

Leibniz-Institut für Festkörper- und Werkstoffforschung Dresden

Follow this and additional works at: https://digitalcommons.unl.edu/physicsstreubel

Part of the Atomic, Molecular and Optical Physics Commons, Condensed Matter Physics Commons, and the Other Physics Commons

Streubel, Robert; Kronast, F.; Fischer, P.; Schmidt, O. G.; and Makarov, D., "Magnetic soft x-ray tomography of magnetic Swiss roll architectures" (2015). Robert Streubel Papers. 28.

https://digitalcommons.unl.edu/physicsstreubel/28

This Conference Proceeding is brought to you for free and open access by the Research Papers in Physics and Astronomy at DigitalCommons@University of Nebraska - Lincoln. It has been accepted for inclusion in Robert Streubel Papers by an authorized administrator of DigitalCommons@University of Nebraska - Lincoln. 


\title{
Magnetic soft x-ray tomography of magnetic Swiss roll architectures
}

\author{
R. Streubel, ${ }^{1}$ F. Kronast, ${ }^{2}$ P. Fischer ${ }^{3,4}$ \\ O. G. Schmidt,1,5 \& D. Makarov ${ }^{1}$
}

1 Institute for Integrative Nanosciences, IFW Dresden, Dresden, Germany;
2 Helmholtz- Zentrum Berlin für Materialien und Energie GmbH, Berlin,
Germany;
3 Lawrence Berkeley National Laboratory, Berkeley, CA;
4 UC Santa Cruz, Santa Cruz, CA;
5 TU Chemnitz, Chemnitz, Germany

A further increase in performance of state-of-the-art spin-electronics can be achieved by either miniaturizing its functional components or harnessing the third dimension. The first route, however, faces physical limitations as properties of nanostructures and their response to external stimuli can drastically change in reduced dimensions. An alternative way is to go from planar 2D structures to 3D architectures [1]. Such 3D functional elements can be obtained for example by rolling up initially planar strained thin films into Swiss roll like objects with multiple windings (Fig. 1a). A major advantage of this technology platform is the possibility to fabricate compact multifunctional 3D architectures with film qualities similar to those of planar systems and peculiar properties only occurring in hollow cylindrical objects [2-4]. Their magnetic properties are generally characterized by integral techniques, such as ferromagnetic resonance [2], magneto-robotic probing [3],

Published in 2015 IEEE International Magnetics Conference (INTERMAG). Conference held 1115 May 2015, Beijing, China; Session BE-04.

doi:10.1109/INTMAG.2015.7156673

Published by IEEE. Used by permission. 


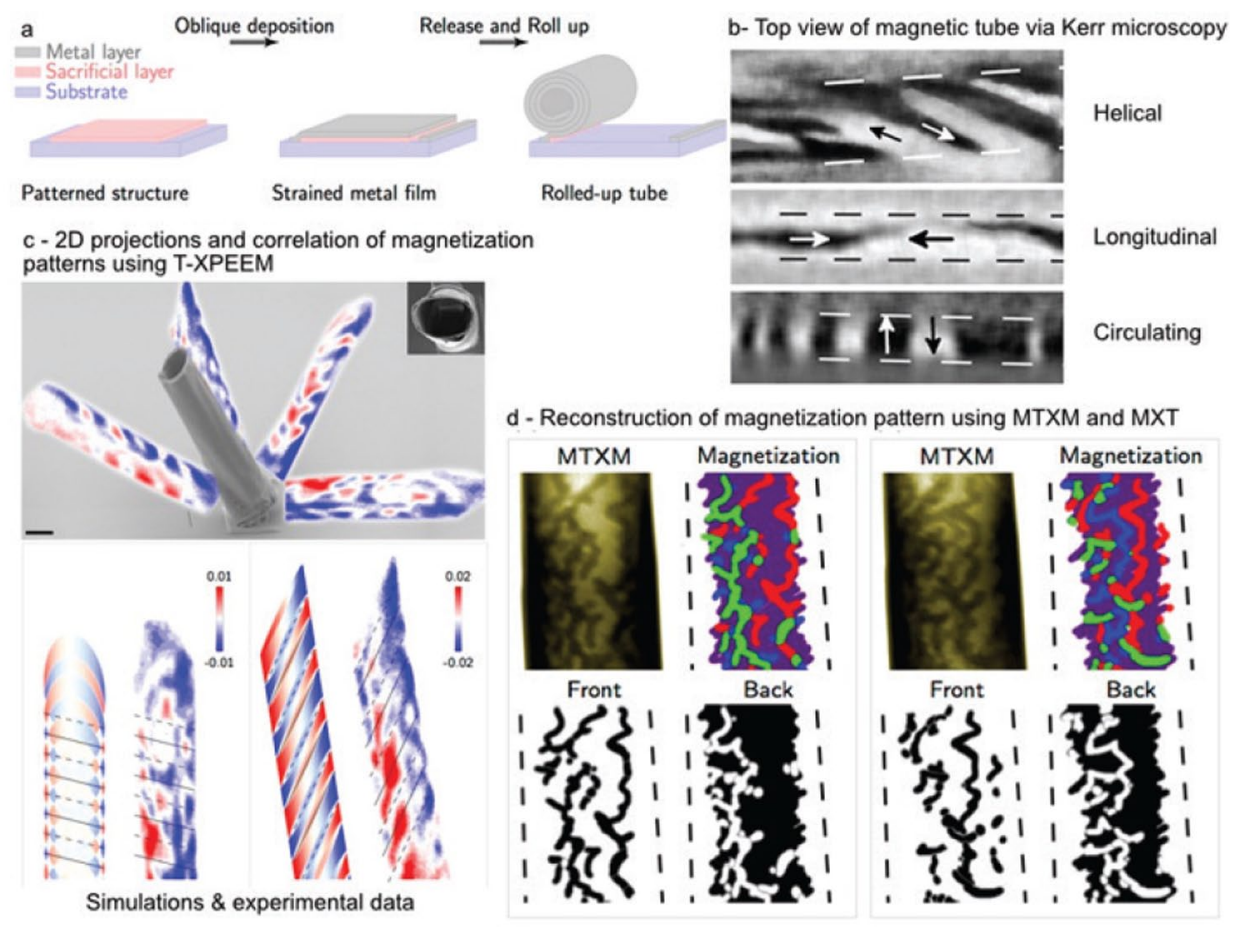

Figure 1

(a) Preparation of curved 3D nanomembranes via rolling up. Depending on magnetostriction constant and shape of the nanomembrane various domain patterns can be set as revealed by Kerr microscopy

(b). A complete characterization of the magnetic domain patterns can only be provided by acquiring 2D projections of the magnetization texture at various projection angles utilizing XMCD as element-specific contrast mechanism.

(c) Transmission XPEEM is applied to identify an azimuthal magnetization within the tightly wound rolled-up nanomembrane based on correlation with XMCD contrast simulations.

(d) Using MTXM together with magnetic X-ray tomography (MXT) algorithms provides means to reconstruct the radial magnetization textures in multiple overlapping curved surfaces.

anisotropic magnetoimpedance/ magnetoresistance $[4,5]$ and cantilever magnetometry [6], or Kerr [7] and x-ray [8,9] microscopies. However, the underlying microscopic magnetization configuration can in general not be retrieved by such means. While already established magnetic neutron tomography [10] and electron holography [11] may be applied to probe either microscopic or nanoscopic samples, a technique 
that provides direct access to the magnetization of 3D-shaped mesoscopic objects with nanometer spatial resolution of the magnetic contrast is highly demanded but not yet developed. Here, we put forth the approach of tomographic imaging of magnetization configurations in 3D-shaped objects relying on x-ray magnetic circular dichroism (XMCD) as element-specific contrast mechanism. A reconstruction of an arbitrary magnetization would require acquiring projections along three orthogonal rotation axes. To minimize experimental affords while exploring the capability of magnetic soft x-ray tomography, we constrain our study to Swiss roll architectures with either in-plane or out-of-plane magnetic anisotropy that demand recording a set of 2D images of the magnetic patterns taken at different angles along a single rotation axis. Swiss roll architectures consisting of in-plane magnetized magnetostrictive Ni thin films possess a residual strain that induces a magnetic easy axis favoring either longitudinal, spiral-like or azimuthal alignment of the magnetization (Fig. 1b) as revealed by top view Kerr microscopy imaging [8]. Furthermore, analysis of the shadow contrast in transmission $x$-ray photoemission electron microscopy (XPEEM) [12] provides further insight into the evolution of the domain patterns across the 3Dshaped objects. We successfully imaged the magnetization configuration of layers inside the 3D magnetic architectures [9]. By acquiring the magnetization patterns at various angles and comparing the results to XMCD absorption calculations (Fig. 1c), we are able to prove unambiguously the presence of the azimuthal domain pattern in the tubular object. This information is not accessible using conventional magnetic imaging relying on the analysis of the $2 \mathrm{D}$ project image. Out-of-plane magnetized Co/Pd multilayers self-assembled into tubes with a diameter of 2-3 mm were investigated with magnetic transmission soft $\mathrm{x}$-ray microscopy (MTXM). Whereas the Swiss roll architectures with a large spacer between the neighboring windings show a weak magnetostatic coupling, tightly connected ones reveal only three different contrast levels and abrupt transitions between them, which indicates mutual switching of the magnetization of different layers. Analyzing the XMCD contrast evolution with varying projection angle, magnetization configurations with feature sizes down to $75 \mathrm{~nm}$ could be reconstructed in each layer. By these means, the impact of the number of windings and of surface angles with respect to the initially applied magnetic field on the domain patterns is studied (Fig. 1d). 
To summarize, we present a novel approach — magnetic soft x-ray tomography - to image 3D-shaped magnetic objects on the example of magnetic Swiss rolls. The approach is based on imaging the magnetic objects at different angles using soft x-ray full field microscopies and interpretation of the acquired XMCD contrast via tomographic reconstruction.

\section{$\Delta \Delta \diamond \Delta \diamond \diamond$}

Supported by DFG (MA 5144/2-1) and ERC under European Union's Seventh Framework program (FP7/2007-2013)/ERC n.306277. PF acknowledges support from BES, MSED of the U.S. Department of Energy (DE-AC02-05- CH11231) and by Leading Foreign Research Institute Recruitment Program (2012K1A4A3053565) through the NRF of Korea funded by Ministry of Education, Science and Technology.

\section{References}

[1] O. G. Schmidt and K. Eberl, Nature (London) 410, 168 (2001).

[2] F. Balhorn, S. Mansfeld, A. Krohn, J. Topp, W. Hansen, D. Heitmann, and S. Mendach, Phys. Rev. Lett. 104, 037205 (2010).

[3] E. J. Smith, D. Makarov, S. Sanchez, V. M. Fomin and O. G. Schmidt, Phys. Rev. Lett. 107, 097204 (2011).

[4] M. Vazquez, J. Mag. Mag. Mat. 226-230, Part 1, 693 (2001).

[5] C. Müller, C. C. Bof Bufon, M. E. Navarro-Fuentes, D. Makarov, D. H. Mosca, and O. G. Schmidt, Appl. Phys. Lett. 100, 022409 (2012).

[6] D. P. Weber, D. Rüffer, A. Buchter, F. Xue, E. Russo-Averchi, R. Huber, P. Berberich, J. Arbiol, A. Fontcuberta i Morral, D. Grundler, and M. Poggio, Nano Lett. 12, 6139 (2012).

[7] R. Streubel, D. Makarov, J. Lee, C. Müller, M. Melzer, R. Schäfer, C. C. Bof Bufon, S.-K. Kim, and O. G. Schmidt, SPIN 03, 1340001 (2013).

[8] R. Streubel, J. Lee, D. Makarov, M.-Y. Im, D. Karnaushenko, L. Han, R. Schäfer, P. Fischer, S.-K. Kim, and O. G. Schmidt, Adv. Mater. 26, 316 (2014).

[9] R. Streubel, L. Han, F. Kronast, A. A. Ünal, O. G. Schmidt, and D. Makarov, Nano Lett. 14, 3981 (2014).

[10] N. Kardjilov et al., Nat. Phys. 4, 399 (2008); I. Manke et al., Nat. Commun. 1, 125 (2010).

[11] E. Dunin-Borkowski et al., Science 282, 1868 (1998); P. A. Midgley and R. E. Dunin-Borkowski, Nat. Mater. 8, 1476 (2009).

[12] R. Streubel, V. P. Kravchuk, D. D. Sheka, D. Makarov, F. Kronast, O. G. Schmidt, and Y. Gaididei, Appl. Phys. Lett. 101, 132419 (2012). 